\title{
Utilizing image recognition technology for foraminiferal assemblage analyses
}
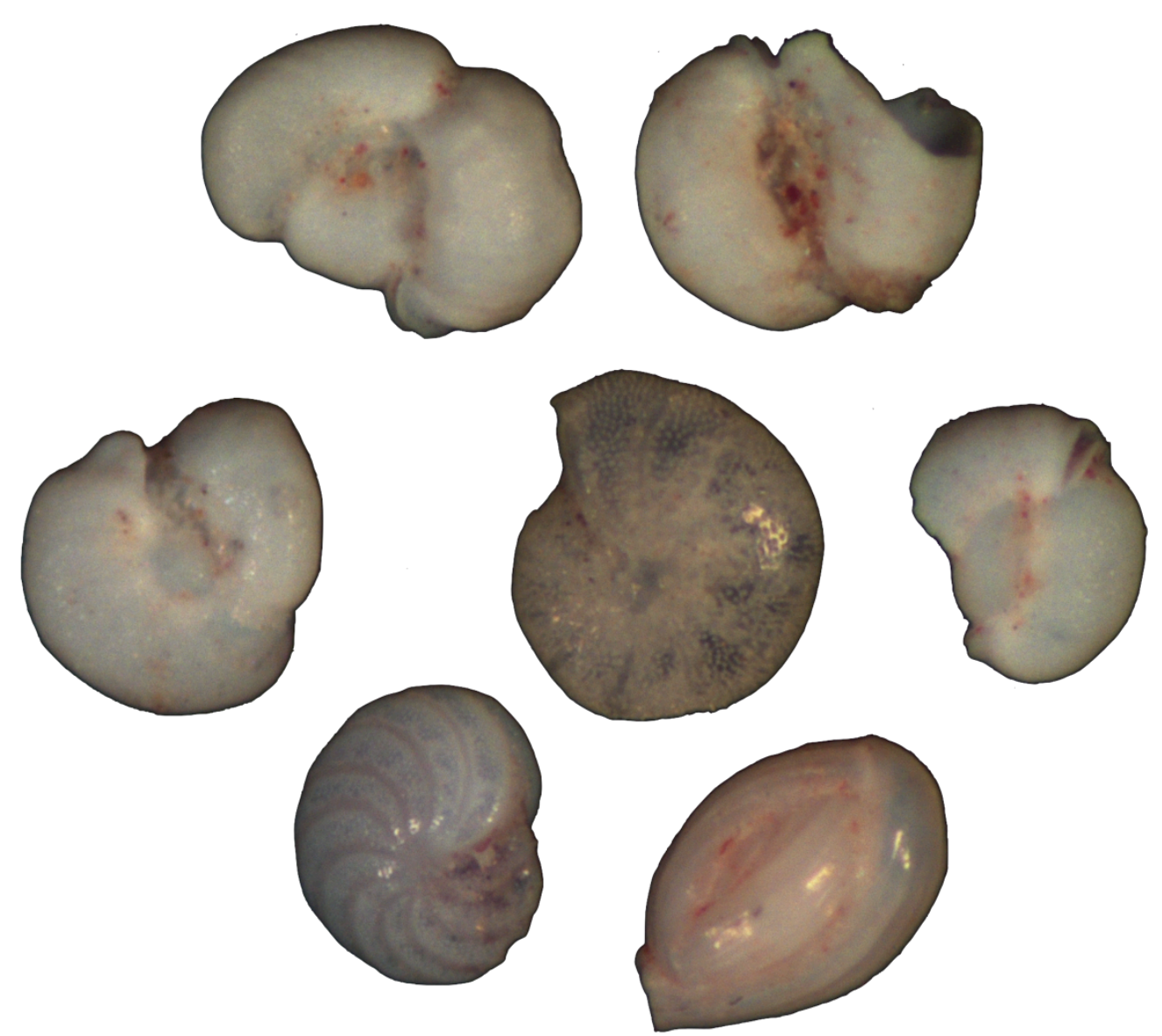

Christian H Gfatter 


\section{Traditional picking}

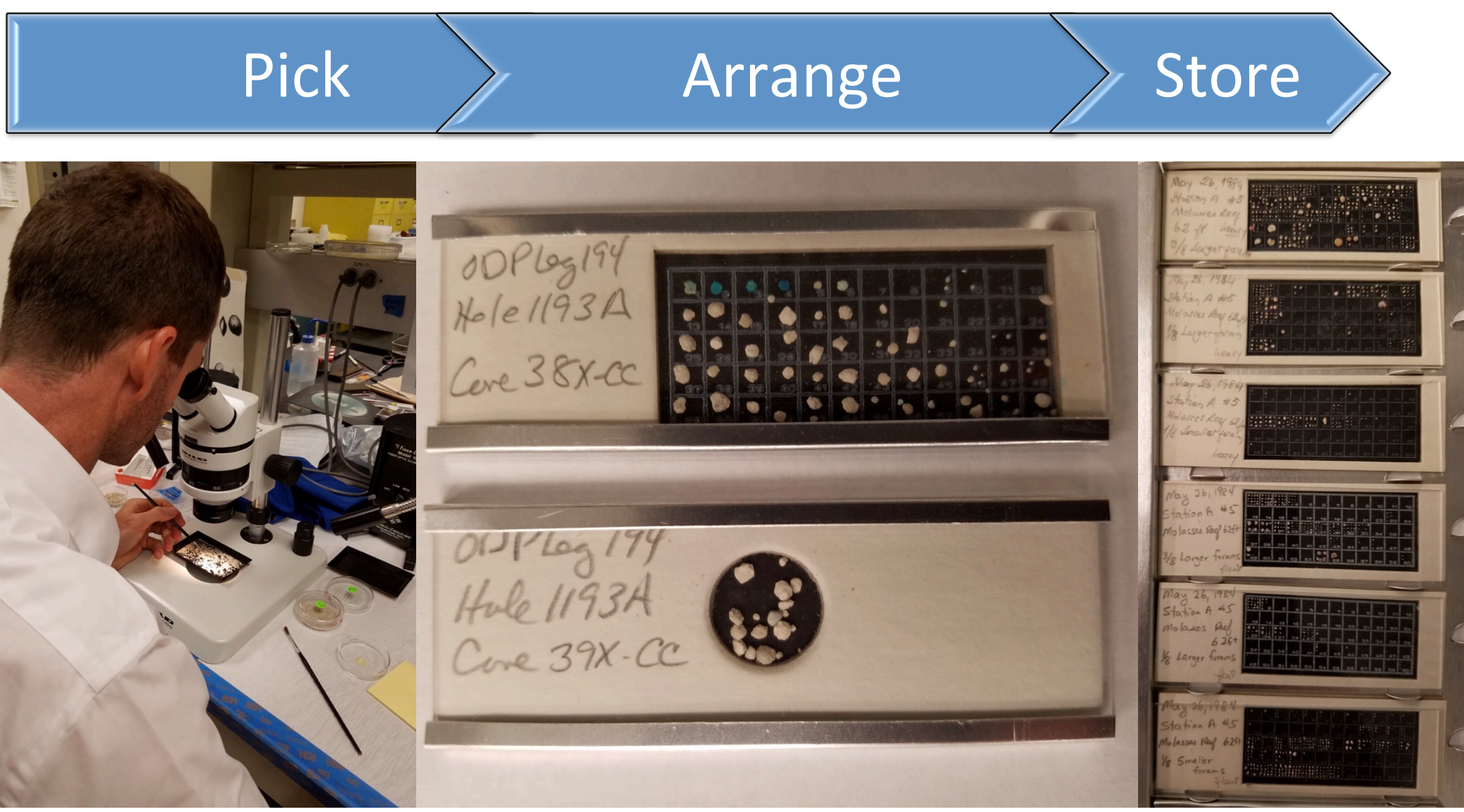




\section{Identification}

\section{Requires skill developed over years}

Novice

\section{Expert}

familiar with $>100$ genera or species

\begin{tabular}{r|r|r|r|}
\hline Identified & using pictures & \multicolumn{1}{|c|}{$\begin{array}{l}\text { using electronic } \\
\text { dichotomous key }\end{array}$} & $\begin{array}{l}\text { using available } \\
\text { resources }\end{array}$ \\
\hline spenera & 57 of 60 & 56 of 60 & 60 of 60 \\
\hline time & 7 of 57 & 14 of 56 & 30 of 60 \\
\hline estimated correct & 2 hours & 3 hours & 1.5 hours \\
\hline
\end{tabular}




\section{The Big Idea}

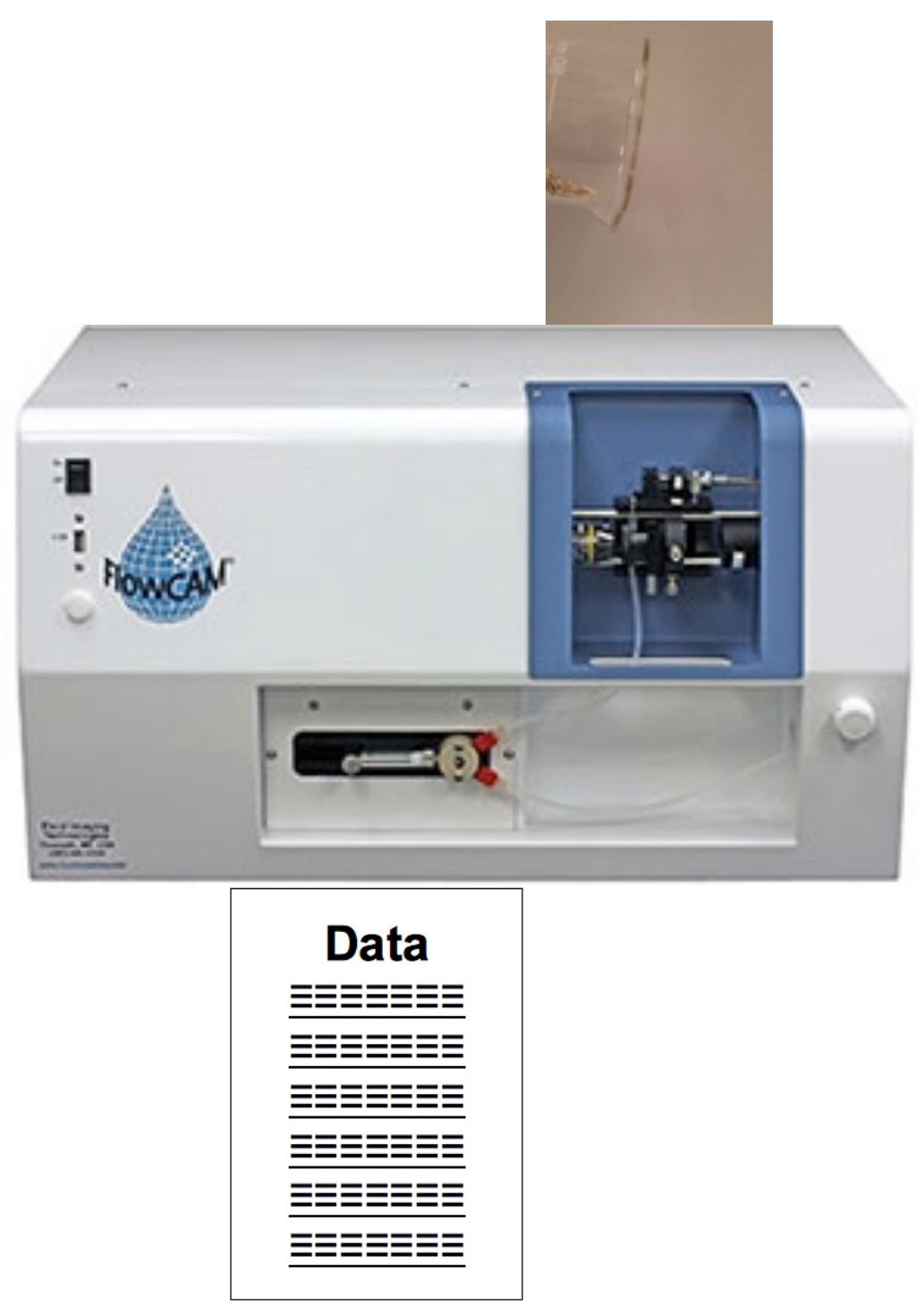

\section{Add sediment containing foraminifera}

\section{Machine sorts \& processes particles}

Software generates data which allows writing detailed Thesis 


\section{Imaging Instruments}

FlowCam ${ }^{\circ}$ VS Series

( $2 \mu \mathrm{m}$ to $2 \mathrm{~mm}$ particles)

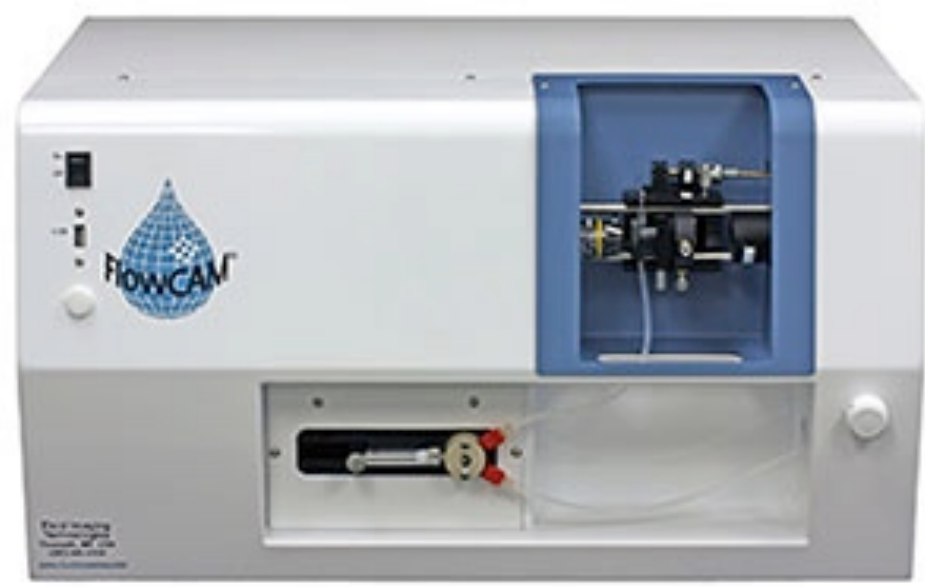

Output from sediment sample

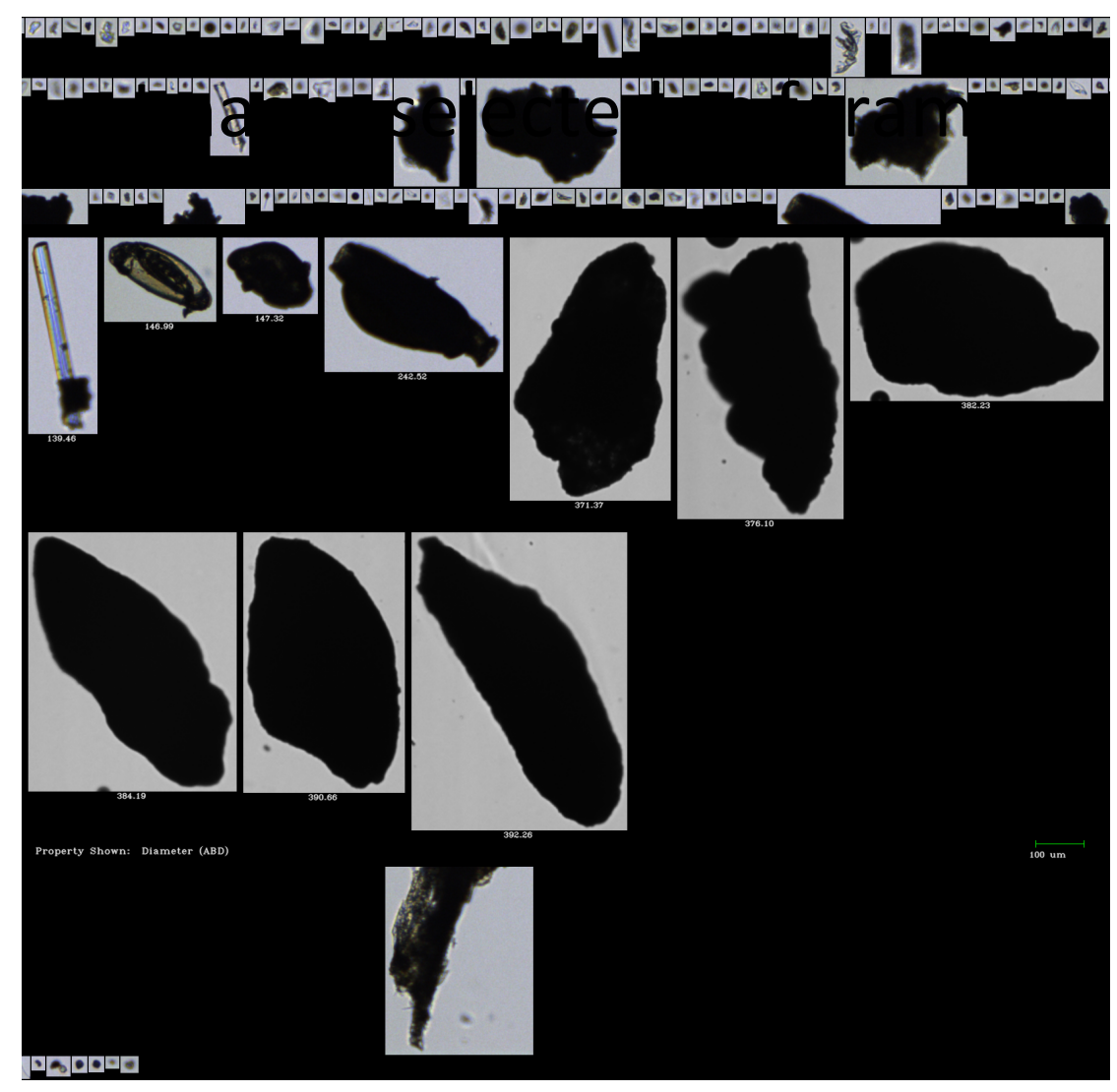




\section{Imaging Instruments}

\section{ZooSCAN}

(liquid zooplankton)

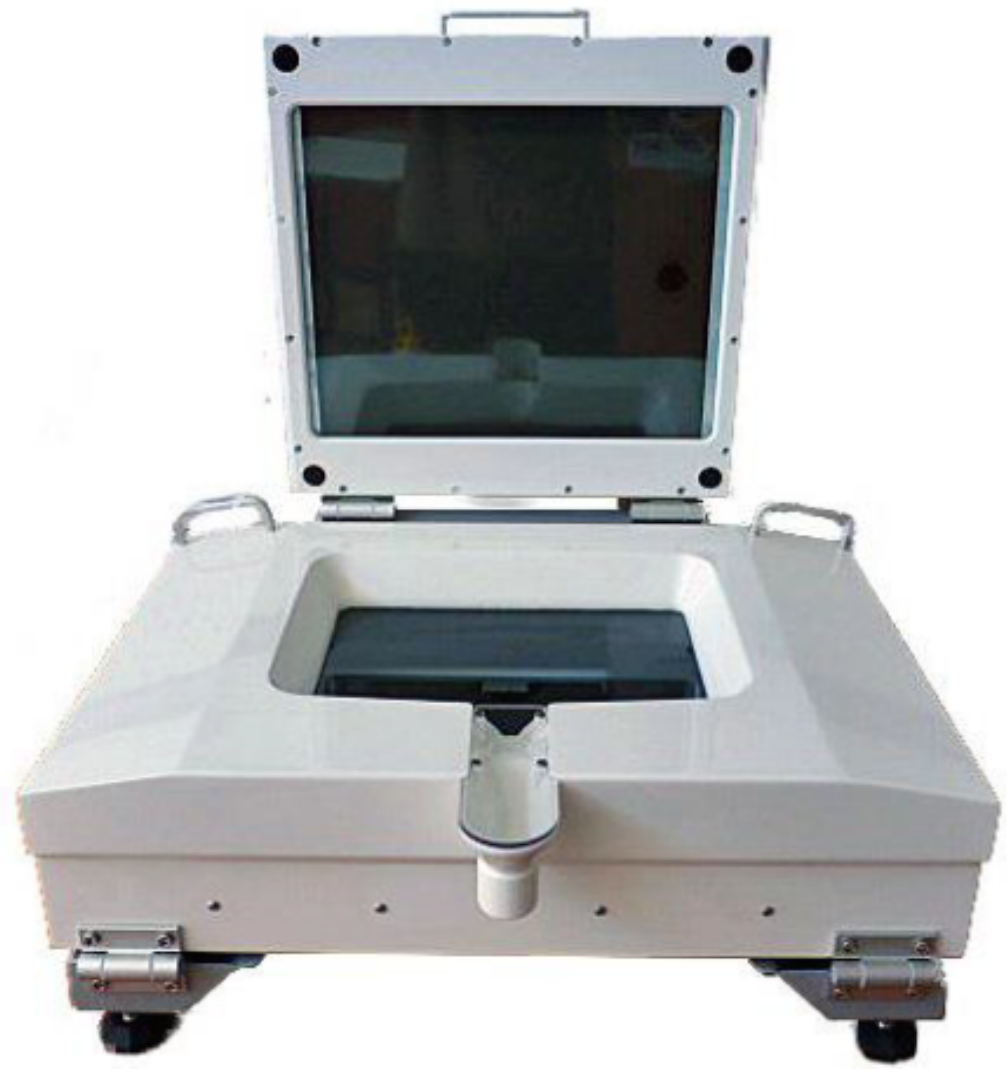

Images of 11 forams

using the lower light 


\section{Imaging Instruments}

\section{ZooSCAN}

(liquid zooplankton)

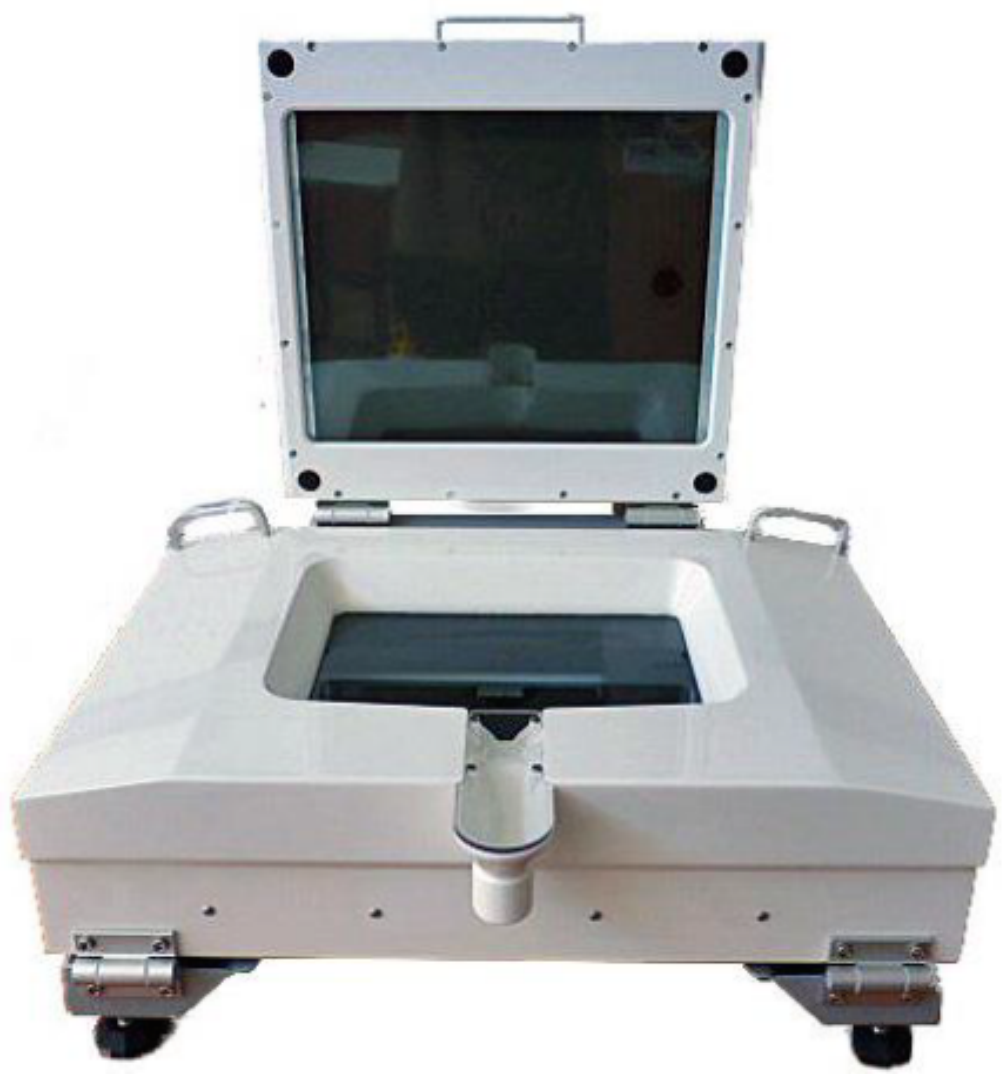

Images of 11 forams

using the upper light 


\section{Imaging Instruments}

\section{Zeiss AxioCam MRc5}

(5 megapixel digital microscope camera)

Archaias angulatus

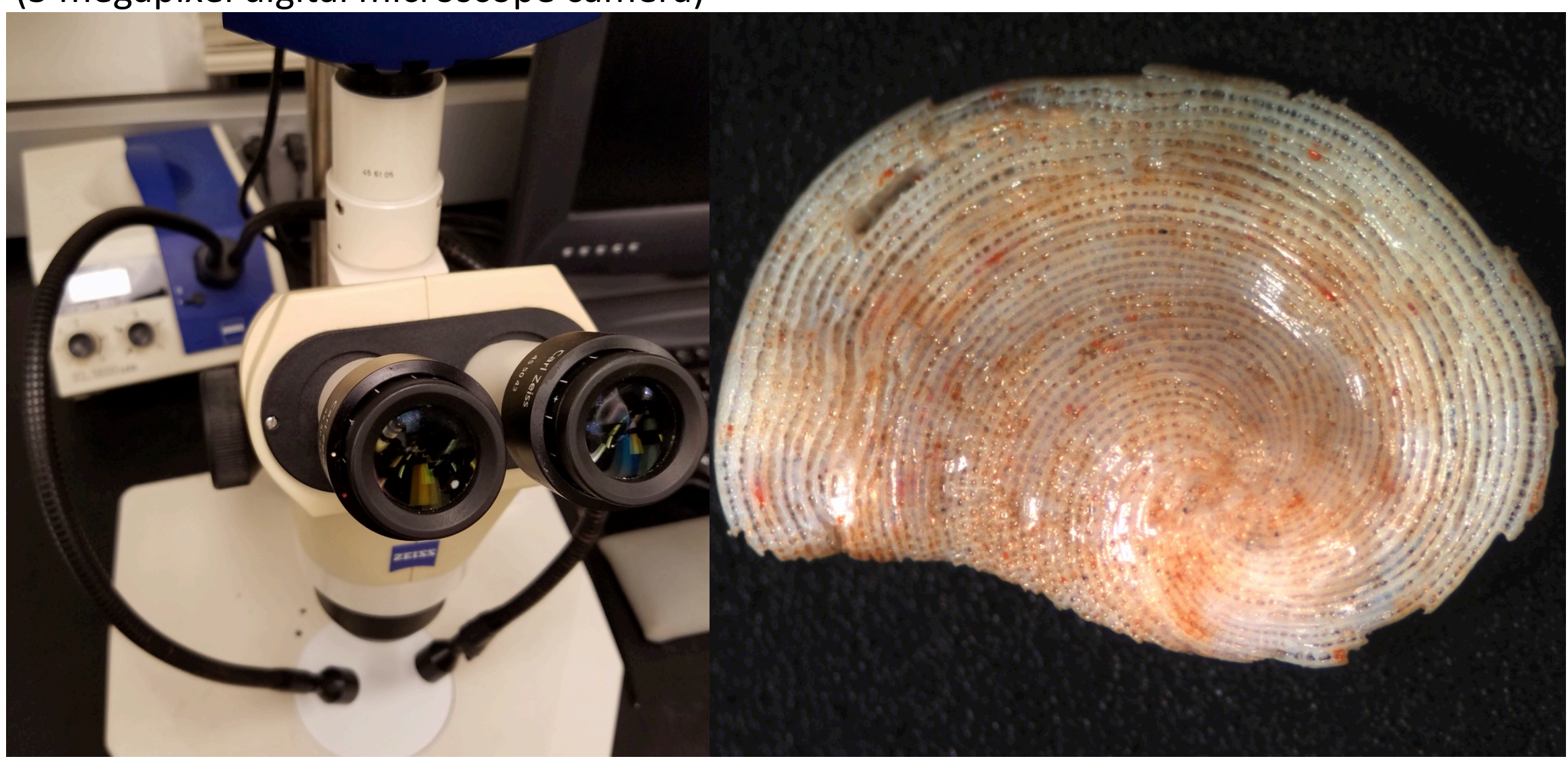




\section{Imaging Instruments}

\section{Zeiss AxioCam MRc5}

(5 megapixel digital microscope camera)

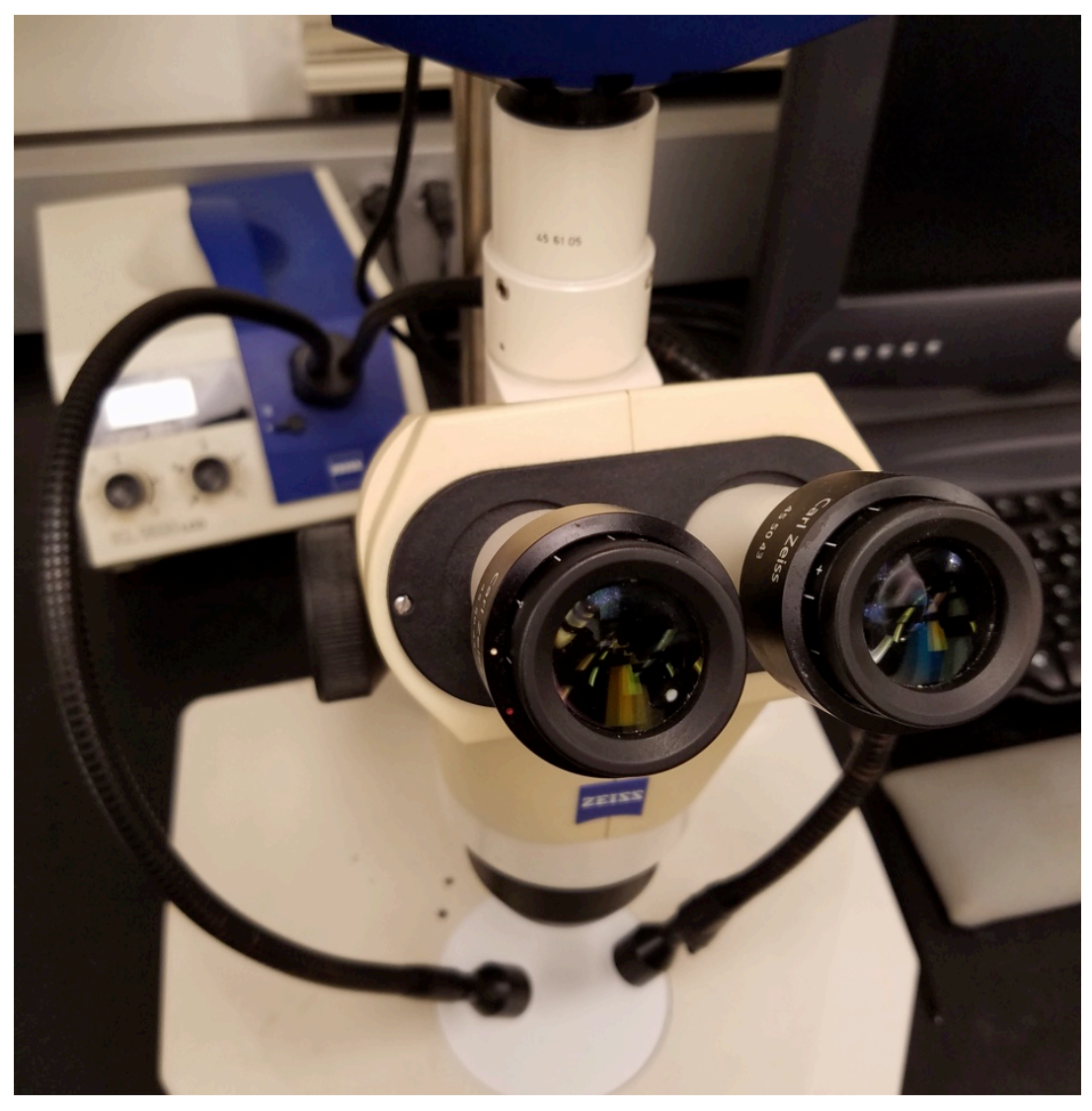

Magnification

\section{$20 x$}

focused on largest foram 


\section{Imaging Instruments}

\section{Zeiss AxioCam MRc5}

(5 megapixel digital microscope camera)

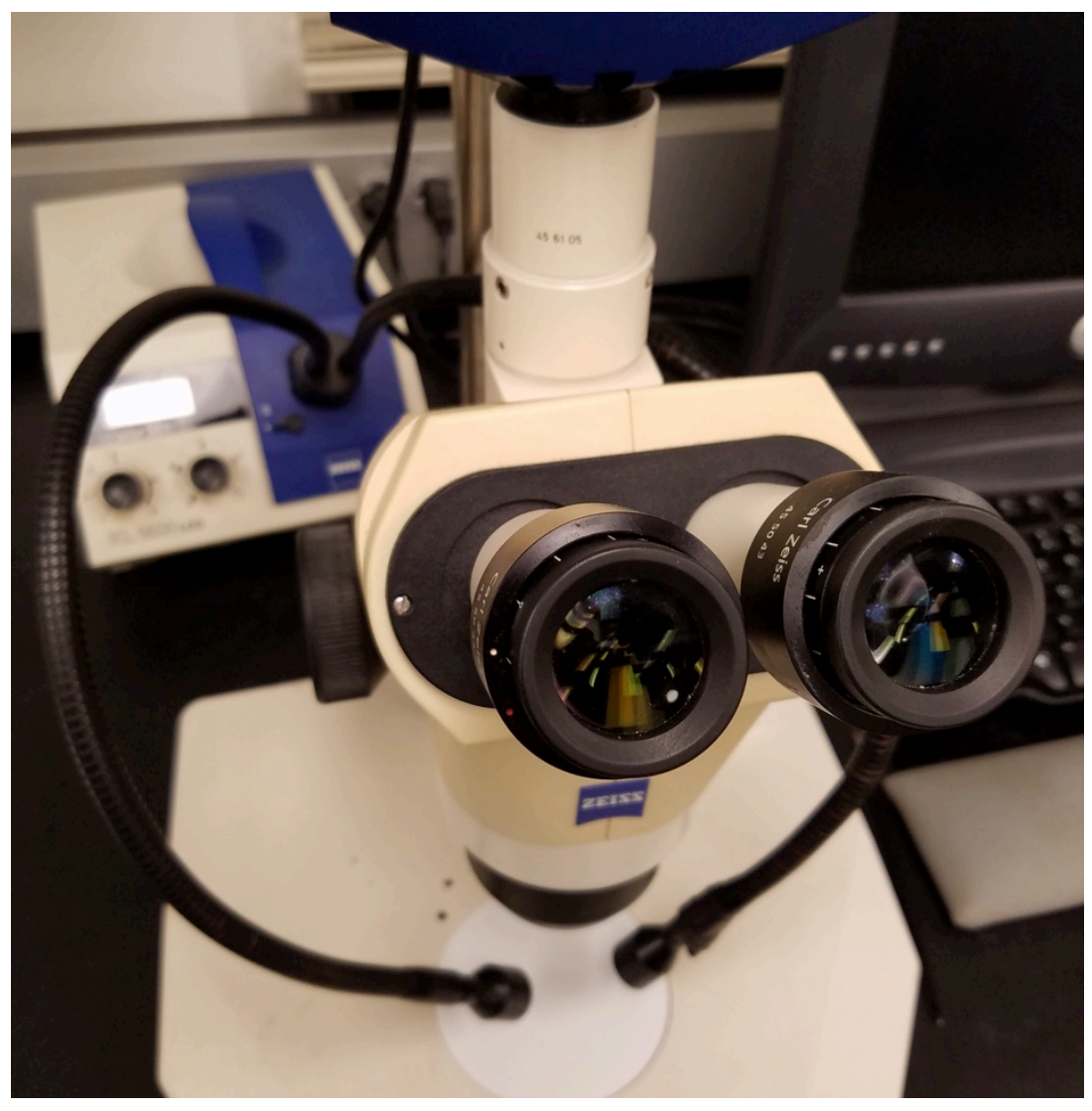

Magnification

\section{$25 x$}

focused on largest foram 


\section{Imaging Instruments}

\section{Zeiss AxioCam MRc5}

(5 megapixel digital microscope camera)

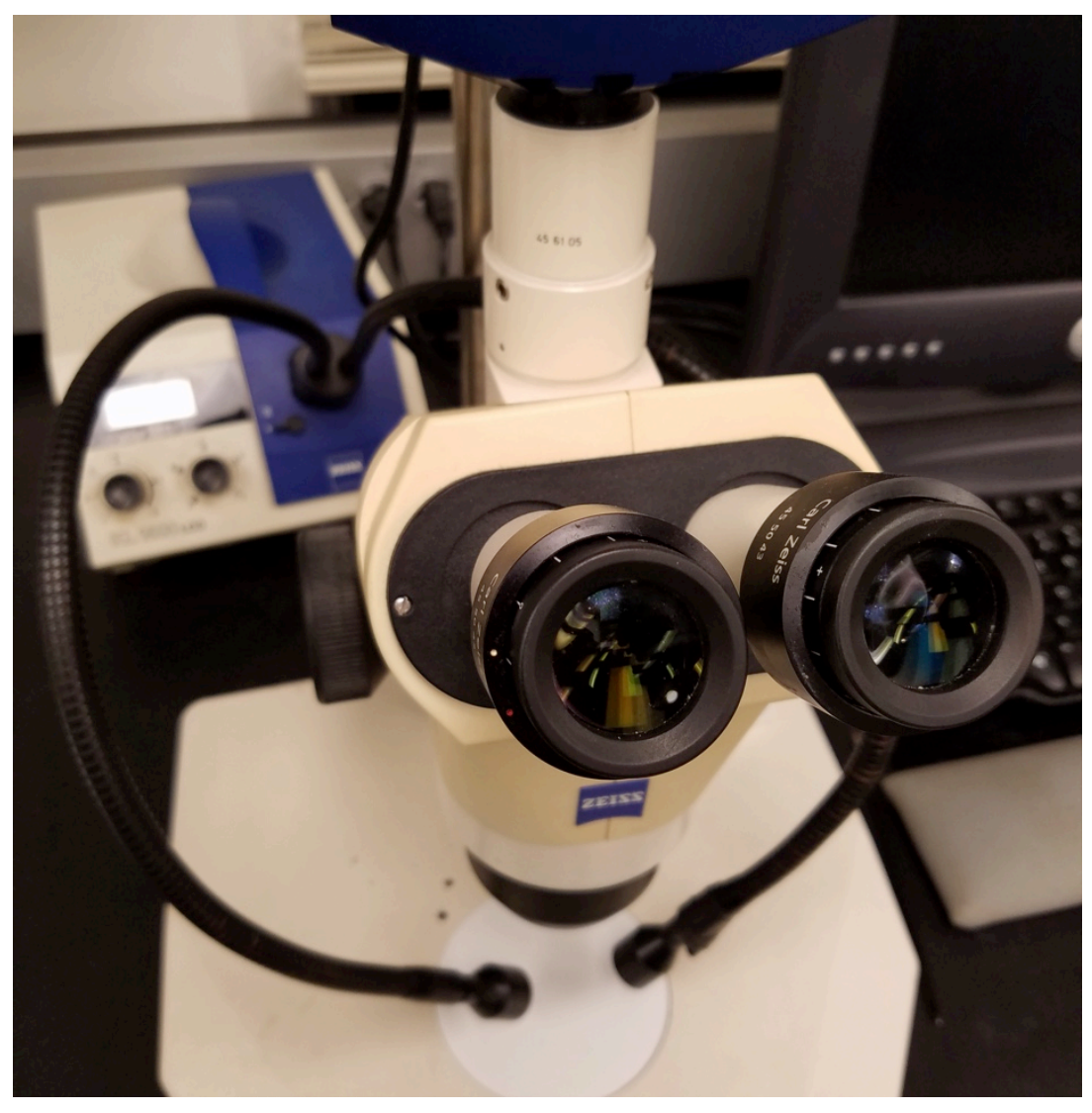

Magnification

\section{$32 x$}

focused on mid-sized foram

○
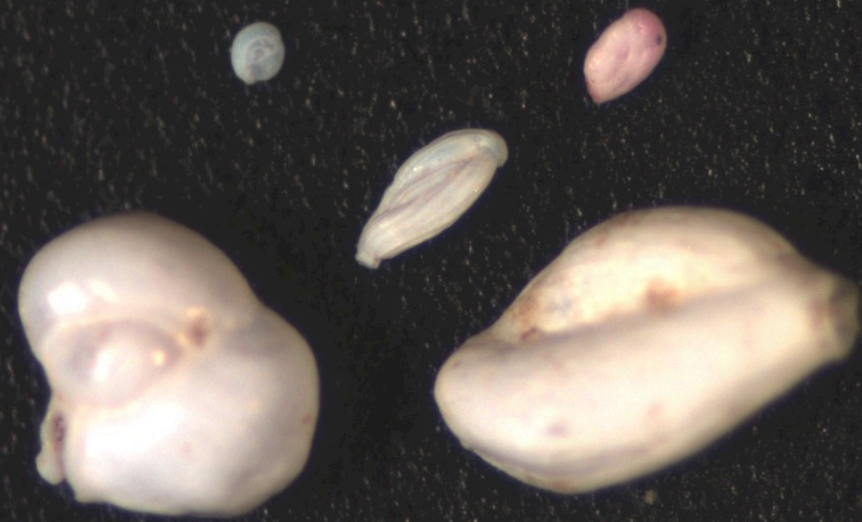


\section{Imaging Instruments}

\section{Zeiss AxioCam MRc5}

(5 megapixel digital microscope camera)

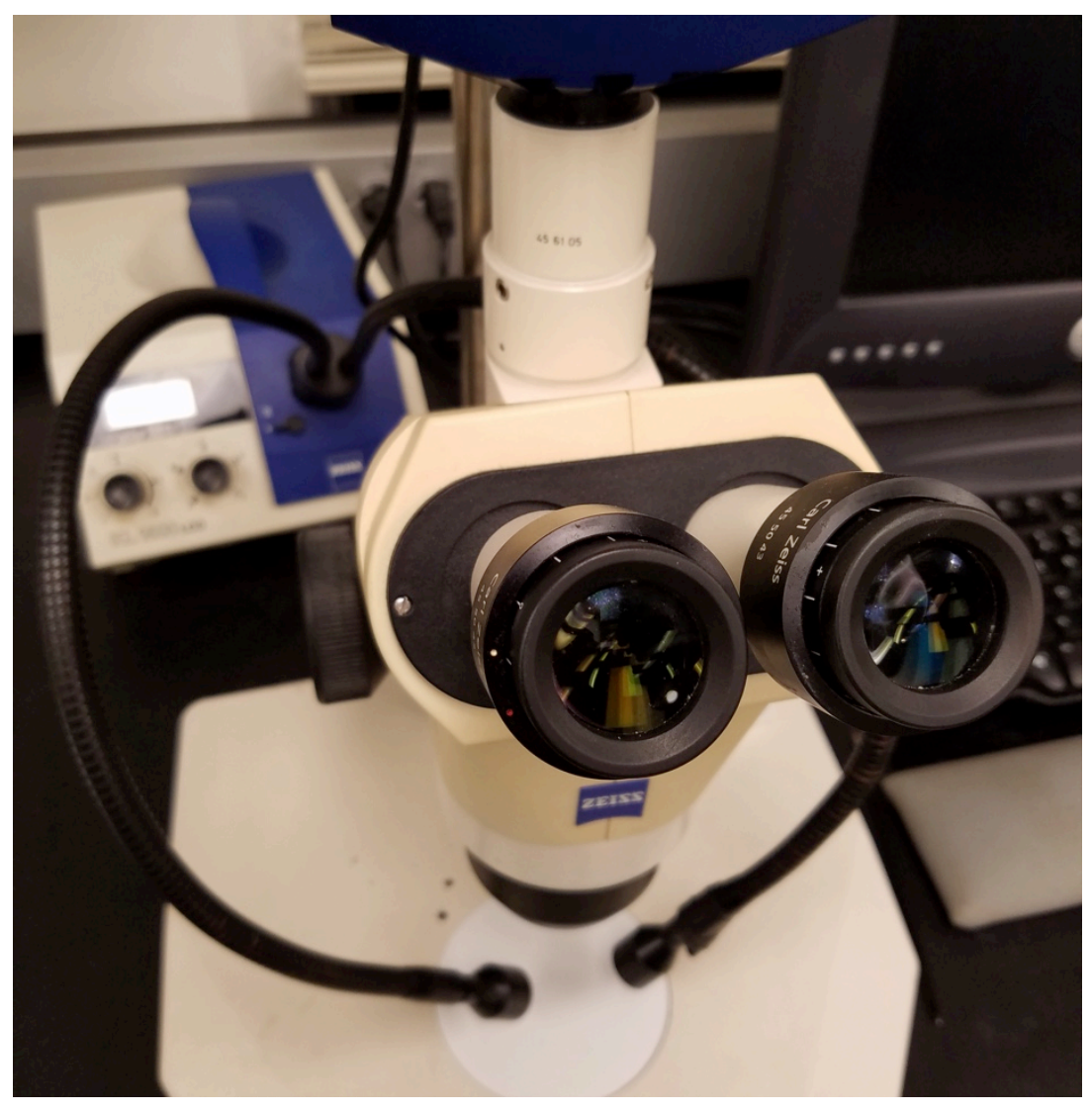

Magnification

\section{$40 x$}

focused on mid-sized foram

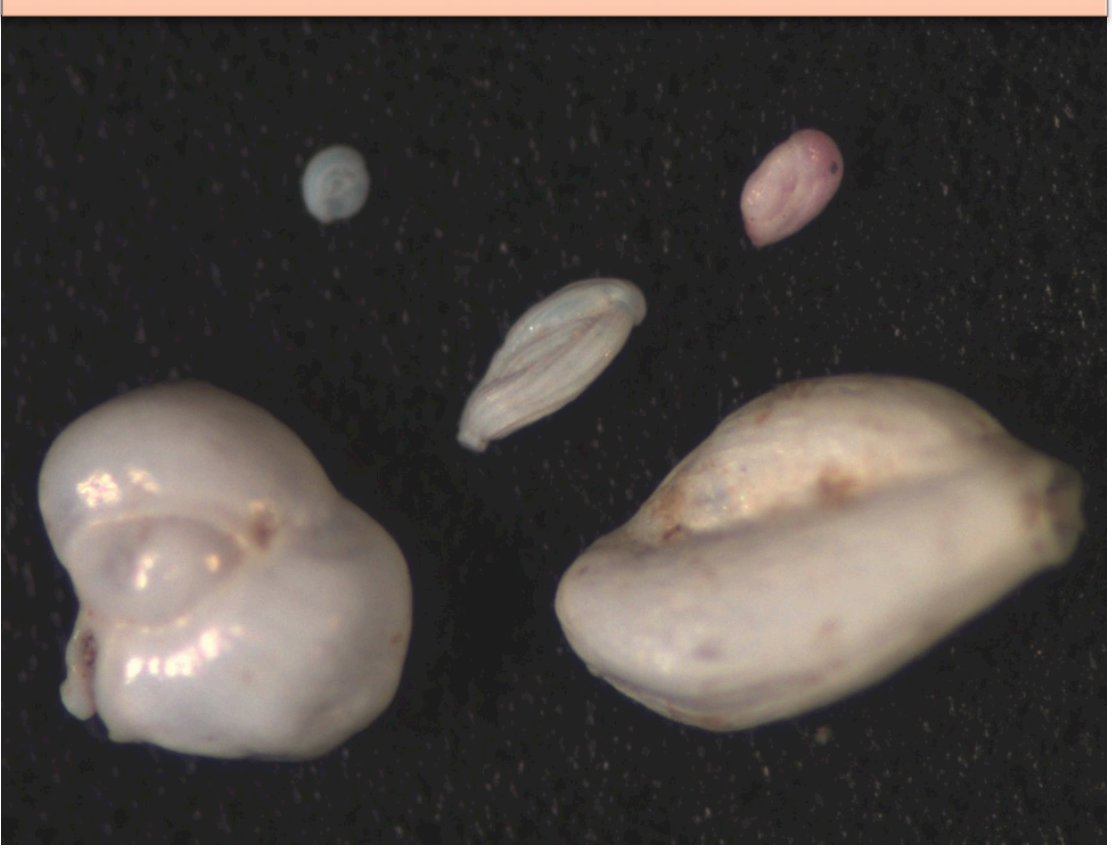




\section{Imaging Instruments}

\section{Zeiss AxioCam MRc5}

(5 megapixel digital microscope camera)

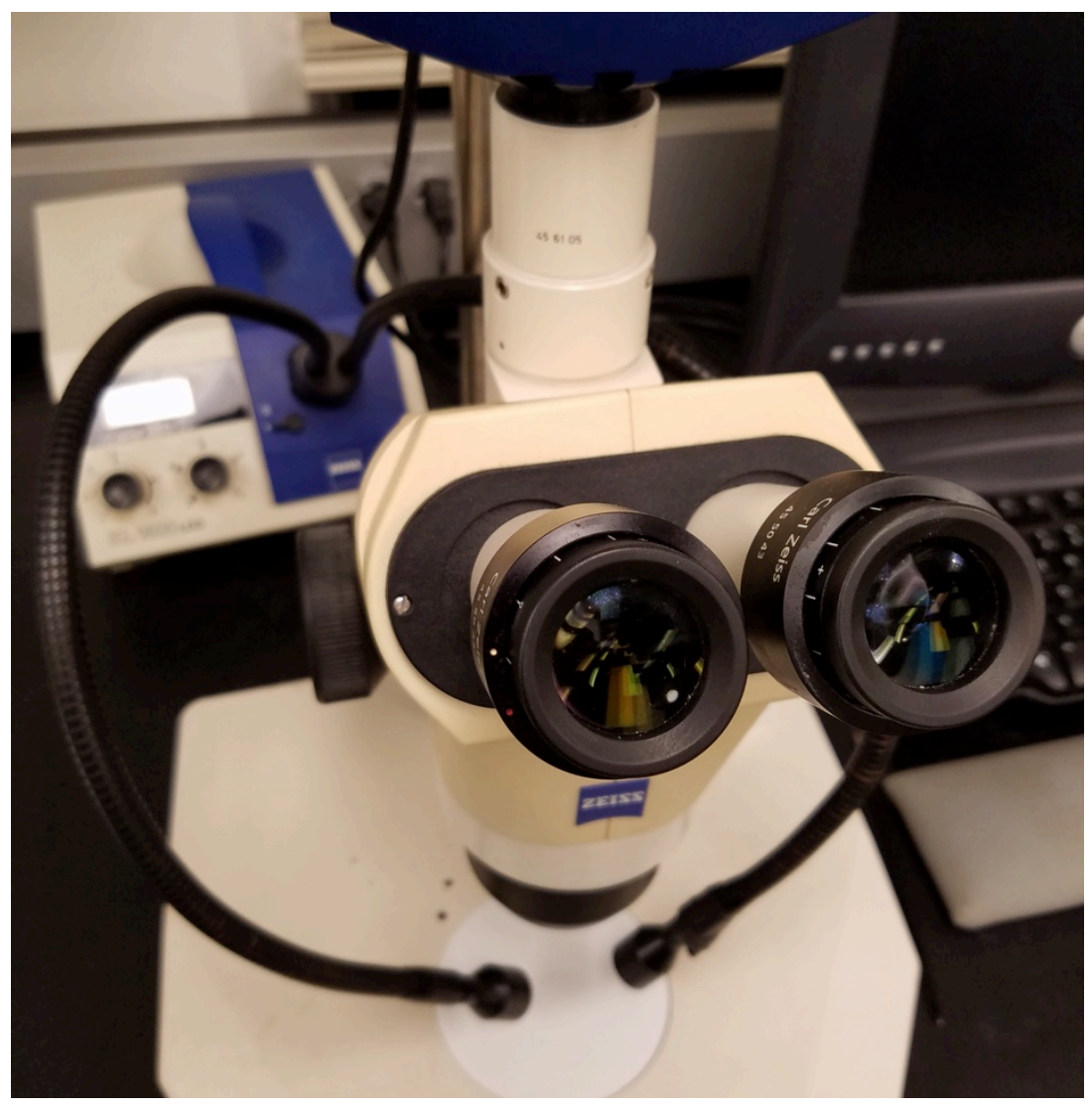

Magnification

\section{$40 x$}

focused on smallest foram

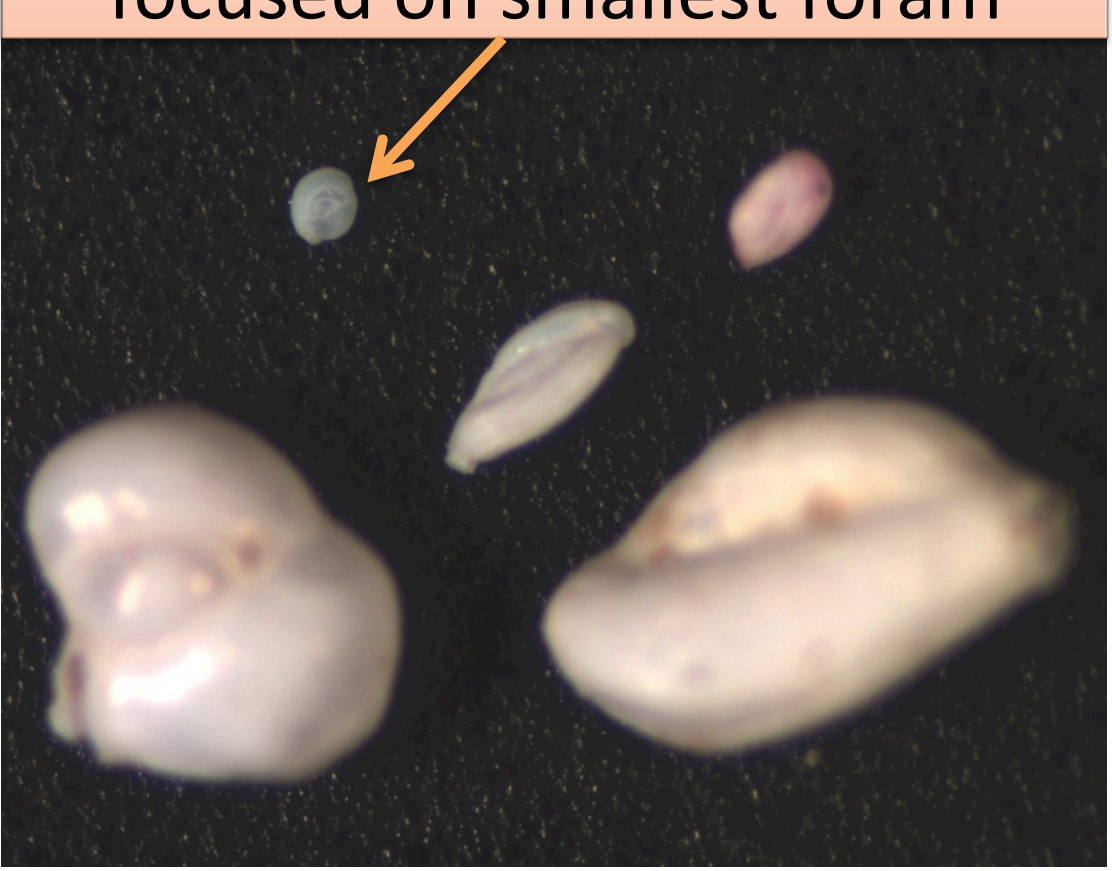




\section{Imaging Instruments}

\section{Zeiss AxioCam MRc5}

(5 megapixel digital microscope camera)

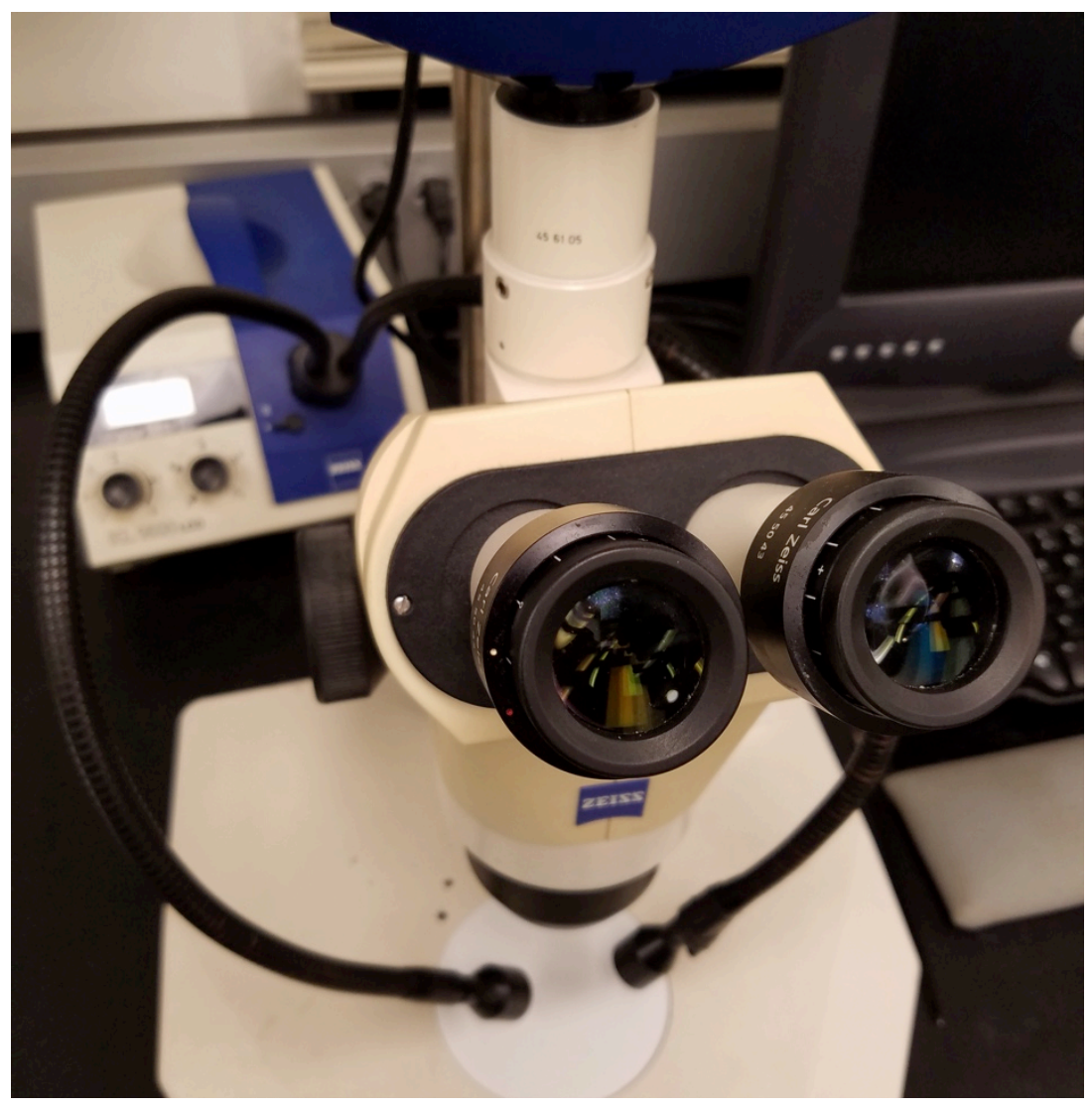

Additional set of images taken for larger specimens

\section{$20 x$}

Archaias angulatus

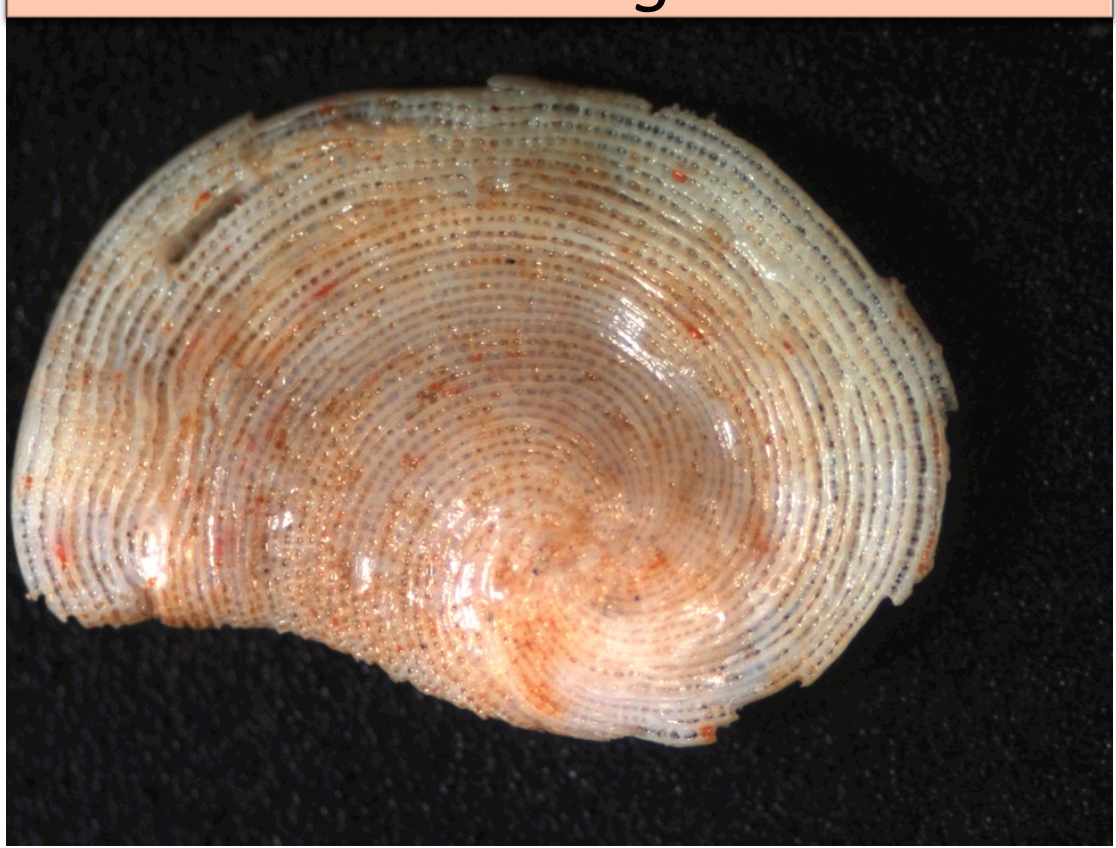




\section{Micropaleoslide arrangement}

Library images from sites

- $1+$ slides per site

- 1 foraminifera per grid

- Several views photographed

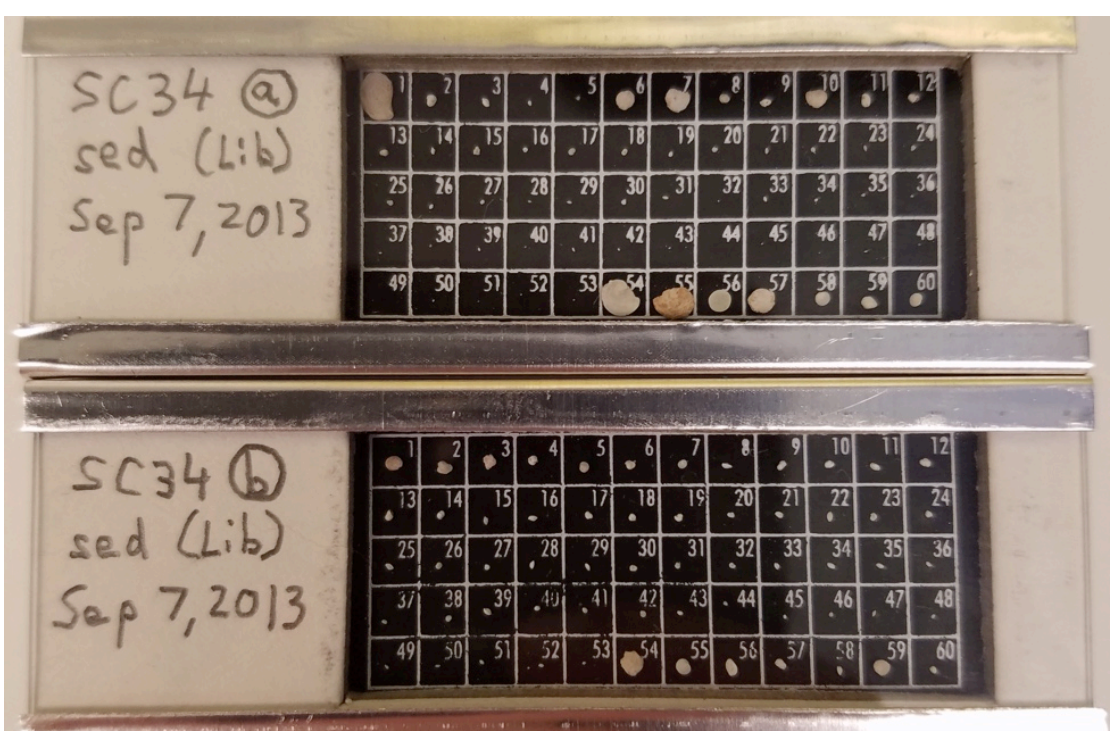

Sampling site images

- 1 slide per site

- $1+$ forams per grid

- $1 / 4$ of grid utilized with forams grouped by size

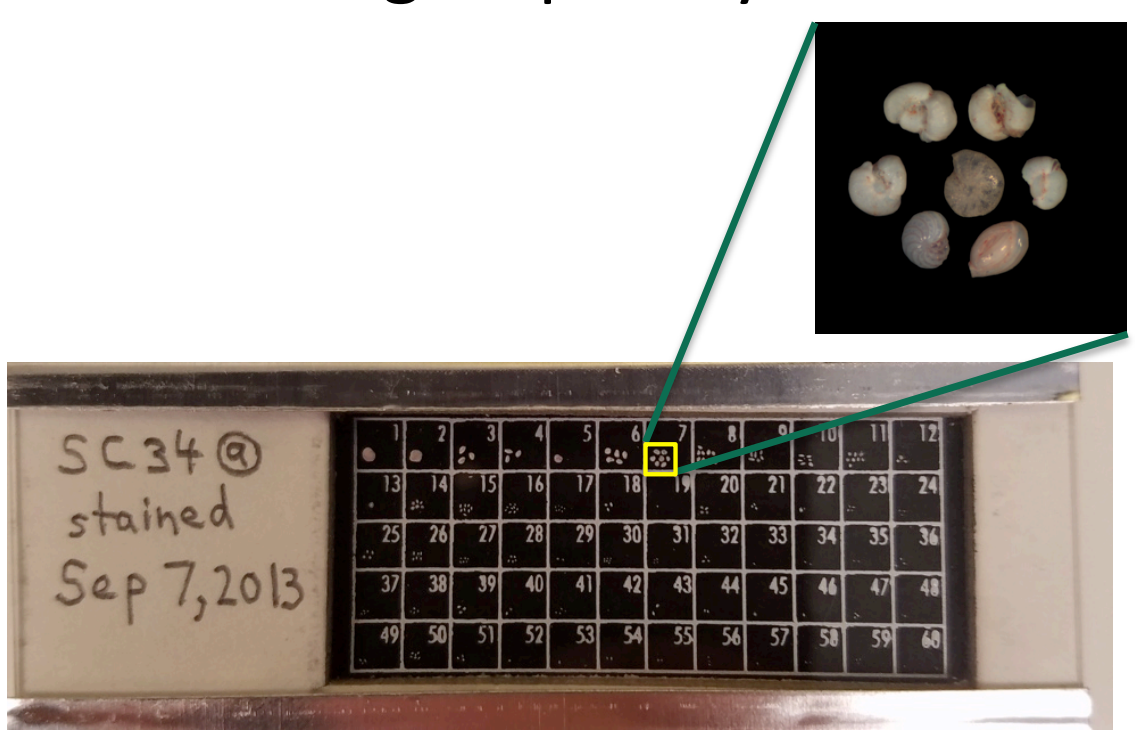


Combining AxioCam pictures with FlowCam image processing software images VisualSpreadsheet
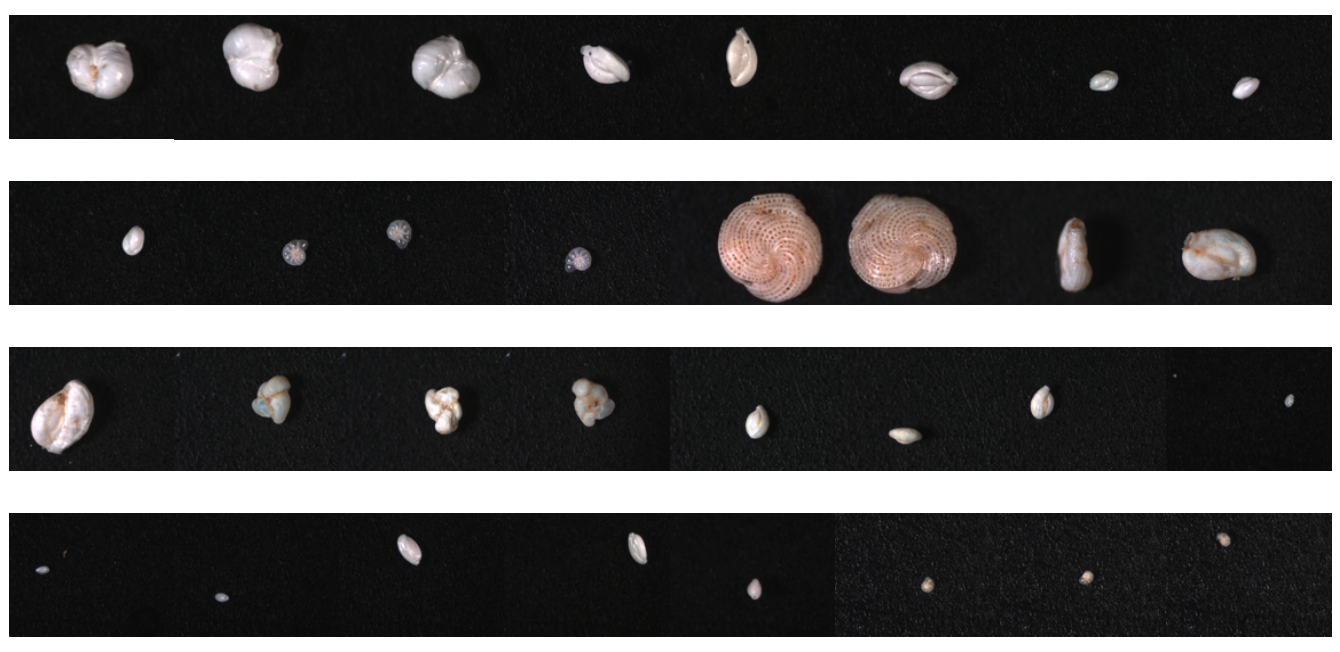

AVI animation

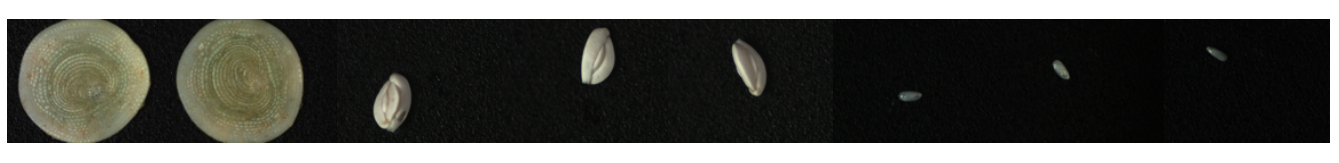




\section{VisualSpreadsheet output}

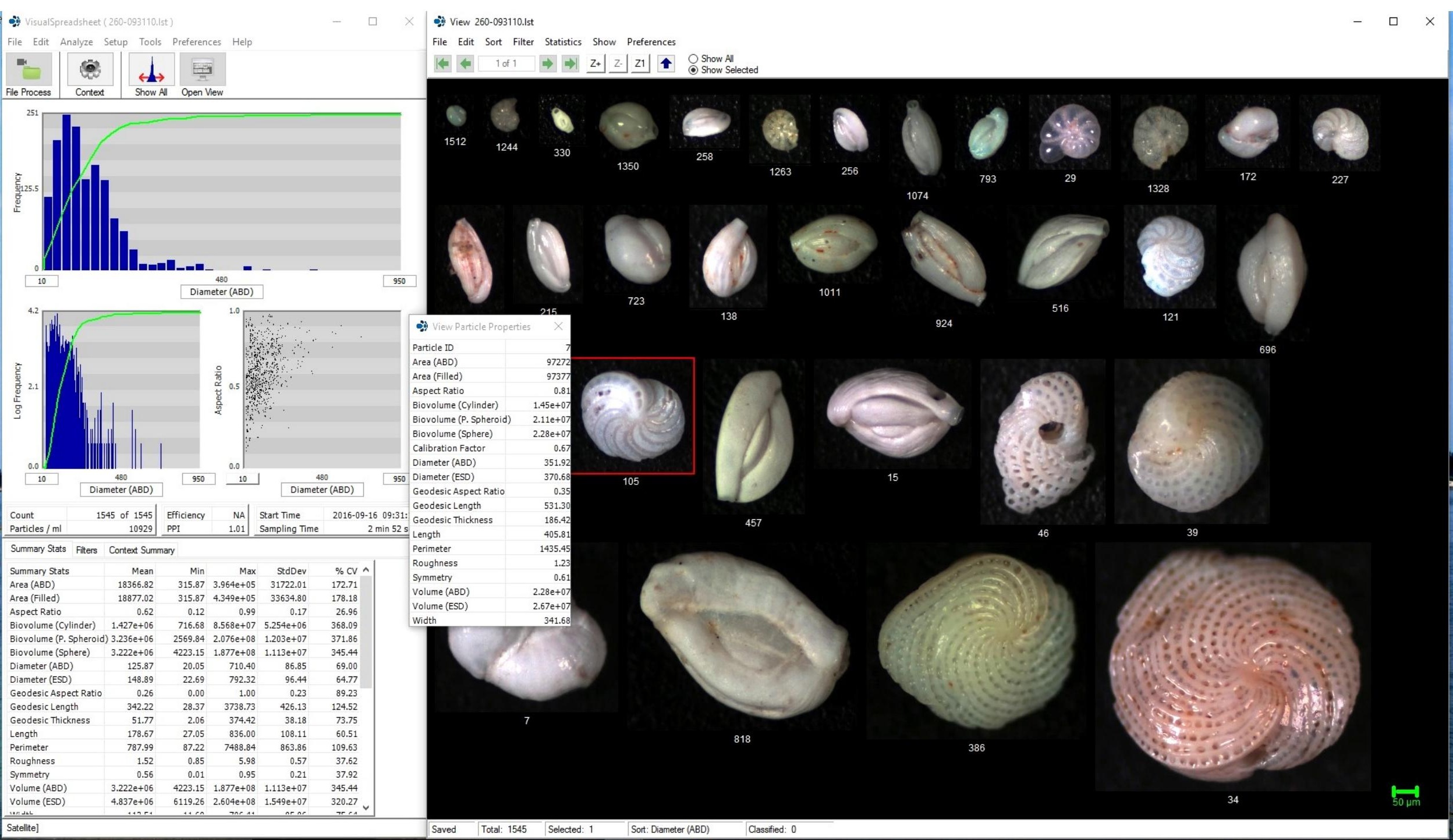




\section{Refinement}

1. Initial classification and measurements systematically made by software

2. Manual post-processing: image rejection, identification overrides, etc.

3. Library grows, helping improve processing of subsequent foraminiferal samples

4. Digital data (images \& records) can be shared and backed-up (physical specimens archived) 


\section{Potential Applications \& Considerations}

- WHAT: heavy mineral sands (e.g., in ancient beach sands), diatoms, ostracods, \& other opaque organisms

- HOW: using trichloroethylene (TCE) separation technique to provide foraminiferal samples for bulk processing

- SOFTWARE: other programs (e.g. SIPPER) may make finer determinations, provide different data, etc. 


\section{Summary}

- Identification efficiency

- Consistency

- Error reduction

- Additional data

- Standardized characterization (e.g. length)

- Digitization

- Accessibility

- Global sharing
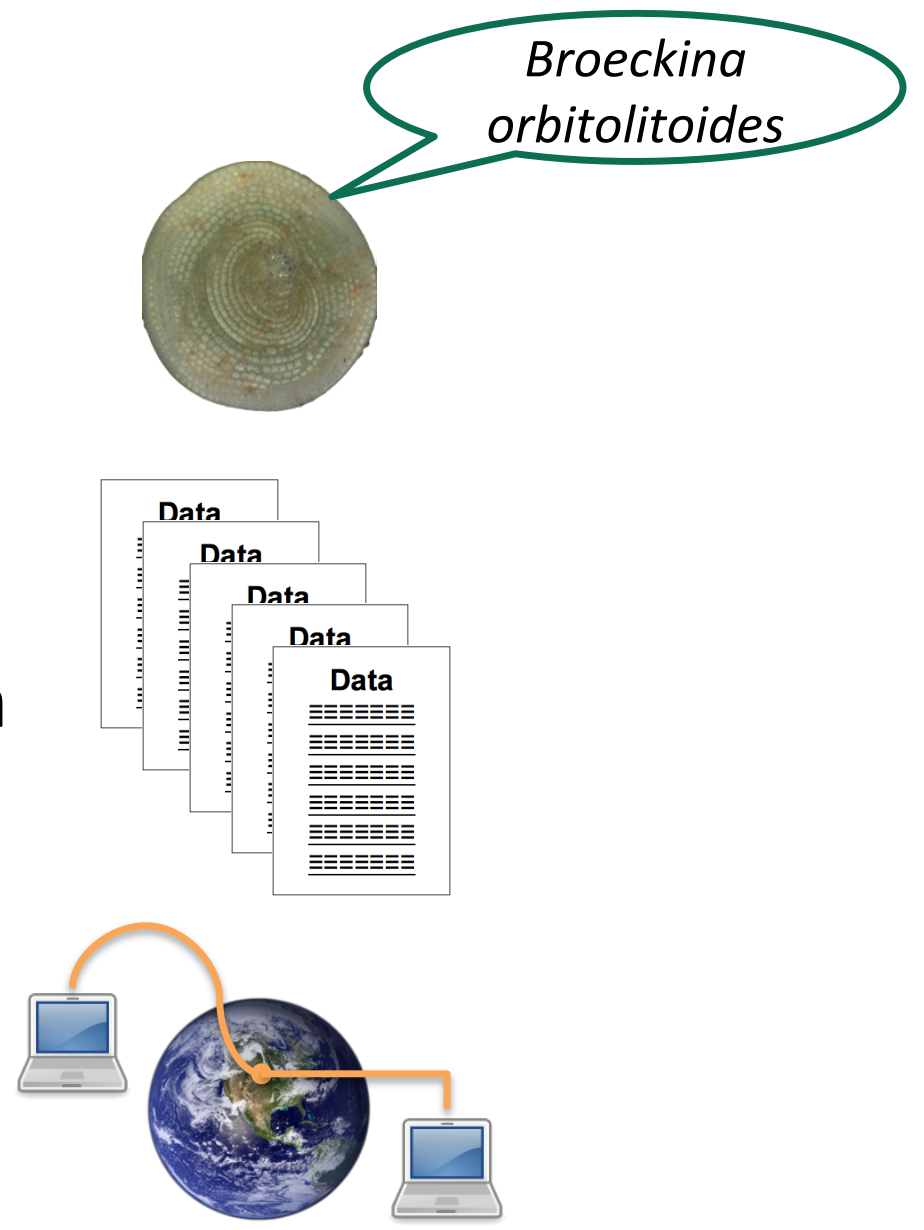


\section{Acknowledgements \& Credits}

- Cushman Foundation for Foraminiferal Research

- Dr. Pamela Hallock Muller

- Kyle Amergian

- Harry Nelson, VP - Aquatic Markets, Fluid Imaging Technologies

- Ian W Bishop, University of Colorado

- Kate Dubickas

- Dr Paul Carlson, Florida Fish and Wildlife Research Institute

Images:

- www.fluidimaging.com/products/flowcam-vs

- www.hydroptic.com/zooscan.html

- commons.wikimedia.org

- cliparts.co

Maps:

- Florida Center for Instructional Technology, University of South Florida 


\section{Questions}
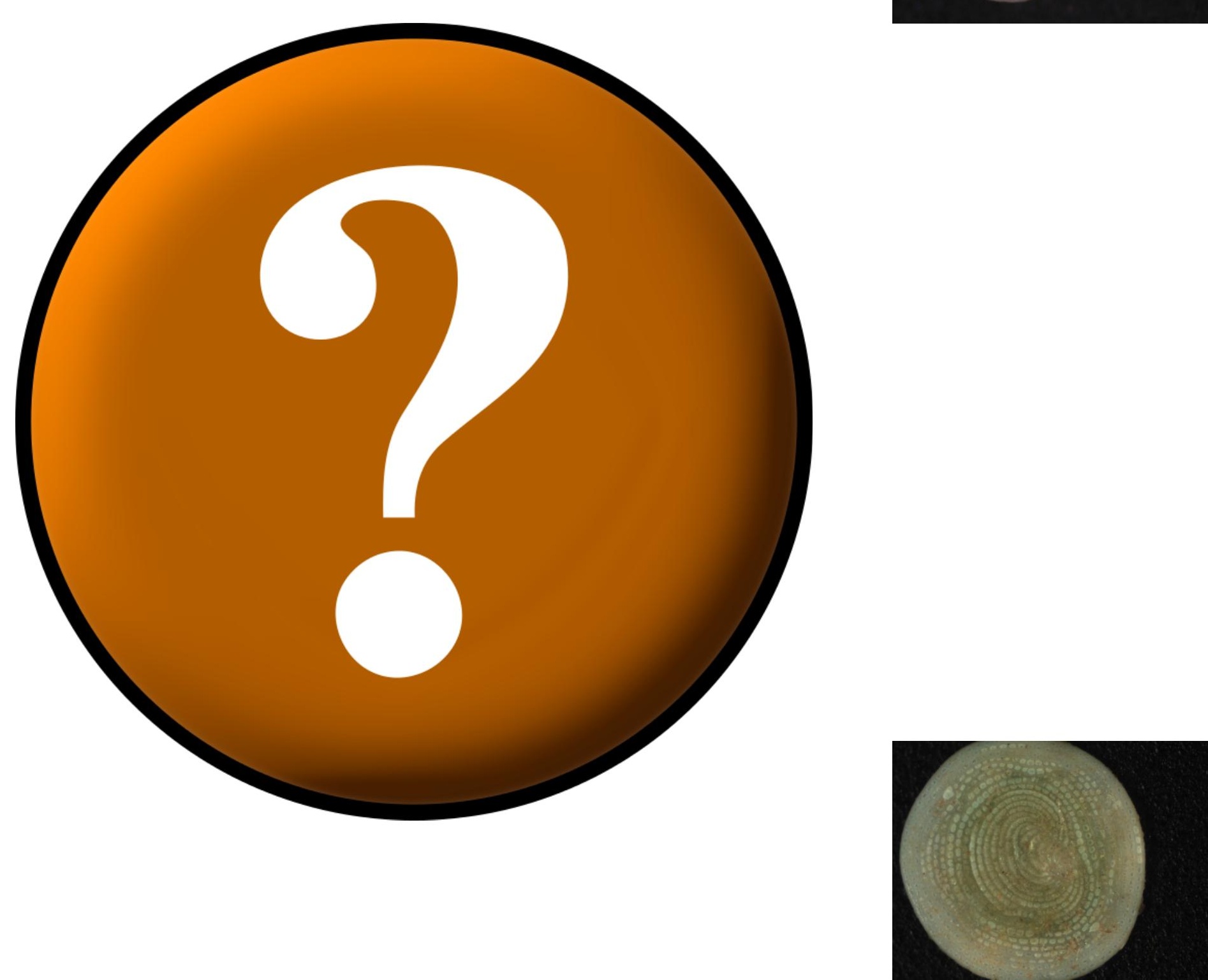


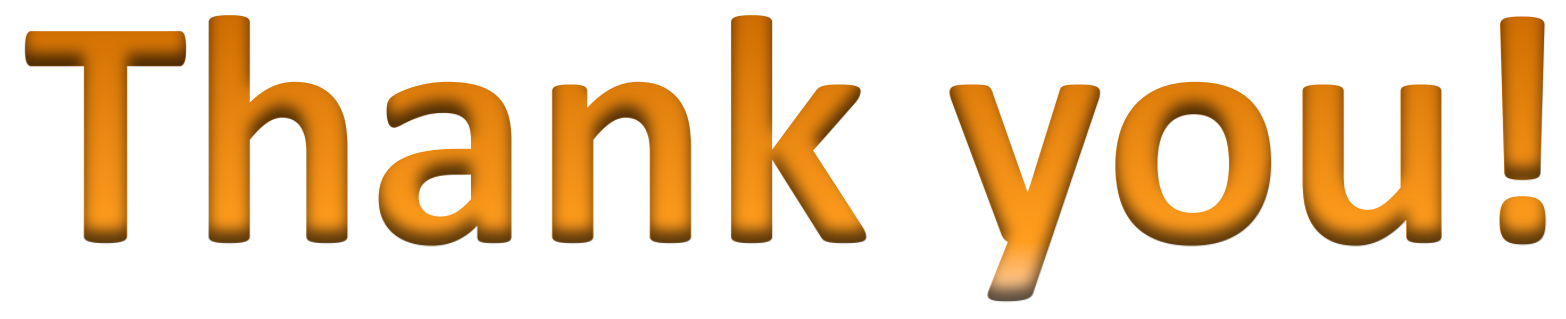

Christian H Gfatter

+1 (312) 321-1777

cgfatter@mail.usf.edu 